UTAP-256

RESCEU-19/97

\title{
Quantum Nucleation of Two-Flavor Quark Matter in Neutron Stars
}

\author{
$\operatorname{Kei~IIDA~}^{(a) *)}$ and Katsuhiko $\operatorname{SATO}^{(a, b) * *)}$ \\ (a) Department of Physics, University of Tokyo, 7-3-1 Hongo, Bunkyo, Tokyo \\ 113 \\ (b) Research Center for the Early Universe, University of Tokyo, 7-3-1 \\ Hongo, Bunkyo, Tokyo 113
}

\begin{abstract}
Rates for nucleation of two-flavor quark matter in a neutron star core, originally composed of nuclear matter in $\beta$ equilibrium, are calculated at zero temperature by a quantum tunneling analysis incorporating the electrostatic energy. We find that a nucleated droplet would develop into bulk matter due to electron screening effects.
\end{abstract}

\footnotetext{
*) E-mail address: iida@utaphp1.phys.s.u-tokyo.ac.jp

**) E-mail address: sato@phys.s.u-tokyo.ac.jp
} 
The possibility that quark matter could exist in neutron stars and the astrophysical consequences have been considered for the past two decades. (See, e.g., Refs. 1)-3).) Usually, the main question was at what pressure the free energy per baryon for electrically neutral quark matter becomes less than that for nuclear matter. Recently, Glendenning ${ }^{1)}$ discovered that, by relaxing the constraint of local charge neutrality, a phase where quark and nuclear matter in $\beta$ equilibrium coexist in a uniform sea of electrons could appear for a finite range of pressures. This is because the presence of strange and down quarks plays a role in reducing the electron Fermi energy and in increasing the proton fraction of nuclear matter. His work ${ }^{1)}$ and the subsequent work by Heiselberg et al. ${ }^{2)}$ claimed that such a mixed phase could exhibit spatial structure such as quark matter droplets embedded in nuclear matter due to surface and Coulomb effects. As the star whose core consists of nuclear matter in $\beta$ equilibrium spins down or accretes matter from its companion star, the central density could become sufficiently large for the mixed phase to be stable. Whether the mixed phase actually nucleates, however, depends on the occurrence of the dynamical processes leading to its nucleation. The first of these processes should be the conversion of nuclear matter to two-flavor quark matter, since the conversion to three-flavor quark matter, which requires many simultaneous weak interactions, is unlikely to occur. ${ }^{4)}$ In this paper, therefore, we consider at what pressure a droplet of two-flavor quark matter forms in nuclear matter and whether it develops into bulk matter or remains finite.

We begin by describing the bulk properties of the various components for densities well above the nuclear saturation density. For nucleons, we write the simple formula for the energy density adopted by Heiselberg et al.:2)

$$
\epsilon_{N}(n, x)=n\left[m_{n}+\frac{K_{0}}{18}\left(\frac{n}{n_{0}}-1\right)^{2}+S_{0}\left(\frac{n}{n_{0}}\right)^{\gamma}(1-2 x)^{2}\right]
$$

where $m_{n}$ is the neutron mass, $n$ is the nucleon number density, $n_{0}=0.16 \mathrm{fm}^{-3}$ is the nuclear saturation density, $x$ is the proton fraction, $K_{0}=250 \mathrm{MeV}$ is the coefficient of the compressional term, and $S_{0}=30 \mathrm{MeV}$ as well as $\gamma=1$ determine the symmetry term. For quarks we adopt the energy density based on the bag model,

$$
\epsilon_{Q}\left(n_{u}, n_{d}, n_{s}\right)=\left(1-\frac{2 \alpha_{s}}{\pi}\right)\left(\sum_{q=u, d, s} \frac{3 \mu_{q}^{4}}{4 \pi^{2}}\right)+B
$$

where $q=u, d$, and $s$ denote up, down, and strange quarks, $n_{q}$ is the number density of $q$ quarks, and $\mu_{q}=\left(1-2 \alpha_{s} / \pi\right)^{-1 / 3}\left(\pi^{2} n_{q}\right)^{1 / 3}$ is the chemical potential of $q$ quarks. In Eq. (2), all quark masses have been taken to be zero, and the QCD fine structure constant and the bag constant have been set as $\alpha_{s}=0.4$ and $B=120 \mathrm{MeV} \mathrm{fm}^{-3}$. The energy density of the 
electrons, which are relativistically degenerate, is $\epsilon_{e}\left(n_{e}\right)=\mu_{e}^{4} / 4 \pi^{2}$ with the electron number density $n_{e}$ and the electron chemical potential $\mu_{e}=\left(3 \pi^{2} n_{e}\right)^{1 / 3}$. We neglect the existence of hyperons and muons.

At a given pressure $P$, we have calculated the baryon chemical potentials for the three bulk phases being electrically neutral. The first phase consists of nuclear matter in $\beta$ equilibrium: $\mu_{n}=$ $\mu_{p}+\mu_{e}$ with the neutron (proton) chemical potential $\mu_{n}\left(\mu_{p}\right)$. The second phase is composed of $u$ and $d$ quark matter arising via deconfinement from the above nuclear matter of proton fraction $x_{\text {eq }}$ so as to satisfy $n_{u} / n_{d}=\left(1+x_{\mathrm{eq}}\right) /\left(2-x_{\mathrm{eq}}\right)$. Lastly, for comparison, we consider a phase containing $u, d$, and $s$ quark matter in $\beta$ equilibrium: $\mu_{d}=\mu_{u}+\mu_{e}$ and $\mu_{d}=\mu_{s}$. Figure 1(a) shows the chemical potentials for these three phases obtained as $\mu=(\epsilon+P) / n_{b}$ with the corresponding total energy densities $\epsilon$ and baryon densities $n_{b}$. The crossings denote the transition points from one bulk phase to another, at which the baryon density jumps as can be seen from Fig. 1(b).

Let us now inquire how long it takes a $u$ and $d$ quark matter droplet to form via deconfinement in $\beta$-equilibrium nuclear matter for pressures in the vicinity of the transition point shown in Fig. 1(a). We assume such a droplet to be a sphere macroscopically characterized by its radius $R$ and describe the tunneling behavior of a virtual droplet in the semiclassical approximation. A Lagrangian for the fluctuating droplet may thus be written as ${ }^{5)}$
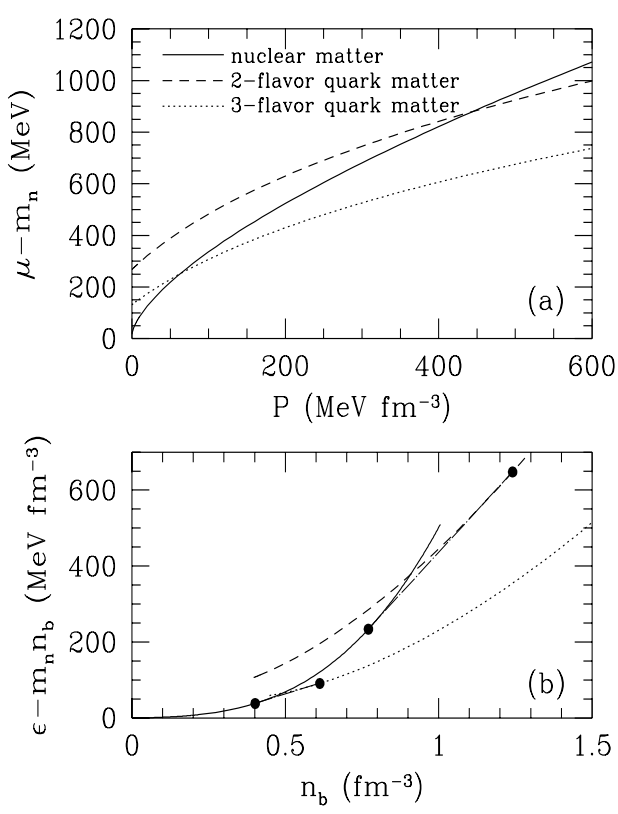

Fig. 1. (a) Chemical potentials for the electrically neutral bulk phases as a function of pressure. The solid line stands for the phase of nuclear matter in $\beta$ equilibrium; the dashed line, the phase of $u$ and $d$ quark matter arising therefrom via deconfinement; the dotted line, the phase of $u, d$, and $s$ quark matter in $\beta$ equilibrium. (b) Energy densities for the corresponding phases as a function of baryon density. The double-tangent constructions (dotdash lines) denote the coexistence of the two bulk phases involved.

$$
L=\frac{1}{2} M(R) \dot{R}^{2}-U(R),
$$

where $M(R)$ is the effective droplet mass, and $U(R)$ is the potential for droplet formation. In the present analysis, no dissipation is considered.

We proceed to evaluate the potential $U(R)$. Due to the high sound velocity of the system $(\sim c)$ it may be assumed that the number density of each component adjusts adiabatically to 
fluctuations of $R$. Consequently, the system retains pressure equilibrium between quark and nuclear matter, and each component, if it makes no contribution to Coulomb screening on the resulting excess droplet charge, is homogeneous, or else is distributed accordingly. Here we assume the quark distributions to be uniform; their shifts due to quark screening ${ }^{6)}$ should reduce the Coulomb energy only slightly. The electron gas is most effective at screening; its Thomas-Fermi (TF) screening length is $\sim 6 \mathrm{fm}$ since $\mu_{e} \sim 300 \mathrm{MeV}$. For the droplet sizes of interest $R \lesssim 10 \mathrm{fm}$, protons, whose TF screening length is estimated from Eq. (1) as $\gtrsim 10 \mathrm{fm}$, may be taken to be uniform. For the initial metastable phase of nuclear matter in $\beta$ equilibrium under pressure $P$, we set the chemical potential as $\mu_{\text {init }}=\left(\epsilon_{\text {init }}+P\right) / n_{\text {init }}$ with the energy density $\epsilon_{\text {init }}=\epsilon_{N}\left(n_{\text {init }}, x_{\text {init }}\right)+\epsilon_{e}\left(n_{e, \text { init }}\right)$, where $n_{\text {init }}, x_{\text {init }}$, and $n_{e, \text { init }}$ give the initial number densities of nucleons and electrons. We can thus express the energy density for the inhomogeneous phase containing a single droplet as the sum of bulk, surface, and Coulomb terms:

$$
\epsilon_{D}=\theta(R-r) \epsilon_{Q}\left(n_{u}, n_{d}, 0\right)+\theta(r-R) \epsilon_{N}\left(n_{\mathrm{init}}, x_{\mathrm{init}}\right)+\epsilon_{e}\left[n_{e}(r)\right]+\epsilon_{S}(r)+\frac{E(r)^{2}}{8 \pi}
$$

where $r$ is the distance from the center of the droplet, $\epsilon_{S}(r)$ is the increase in energy density due to the quark and nucleon distributions in the surface layer whose thickness we assume to be much smaller than $R$, and $E(r)$ is the electric field. We then integrate the difference in the thermodynamic potential per unit volume at chemical potential $\mu_{\text {init }}$ between the initial and inhomogeneous phases over the system volume $V$ which is related to $P$ as $P=$ $-\partial\left(V \epsilon_{\text {init }}\right) / \partial V$. We thus obtain $U(R)$ as

$$
U(R)=\frac{4 \pi R^{3}}{3} n_{b, Q}\left(\mu_{Q}-\mu_{\text {init }}\right)+\Delta E_{e}(R)+4 \pi \sigma R^{2}+E_{C}(R)
$$

where $n_{b, Q}=\left(n_{u}+n_{d}\right) / 3$ is the baryon density inside the droplet, $\mu_{Q}=\left[\epsilon_{Q}\left(n_{u}, n_{d}\right)+\right.$ $\left.\epsilon_{e}\left(n_{e, \text { init }}\right)+P\right] / n_{b, Q}$ is the chemical potential for the uniform quark matter determined by $n_{u}$, $n_{d}, n_{e, \text { init }}$, and $P, \Delta E_{e}(R)=\int_{V} d V\left\{\epsilon_{e}\left[n_{e}(r)\right]-\epsilon_{e}\left(n_{e, \text { init }}\right)\right\}$ is the excess of electron energy over the initial one, $\sigma$ is the surface tension, and $E_{C}(R)$ is the electrostatic energy. In Eq. (5) the curvature energy $(\propto R)$ is omitted. Estimates of the quantities $n_{u}, n_{d}, n_{e}(r), \Delta E_{e}(R)$, $\sigma$, and $E_{C}(R)$ are as follows.

The distribution of electrons has been determined in the linear TF approximation. ${ }^{7)}$ Electrons act to screen the excess droplet charge $Z e=\left(4 \pi R^{3} / 3\right) \rho_{Q}$ with $\rho_{Q}=e\left[\left(2 n_{u}-n_{d}\right) / 3-\right.$ $\left.n_{e, \text { init }}\right]$, and thereby deviate from uniformity in proportion to the electrostatic potential $\phi(r)$ as $\delta n_{e}(r)=n_{e}(r)-n_{e, \text { init }}=\left(\partial n_{e} / \partial \mu_{e}\right)_{\text {init }} e \phi(r)$. The potential $\phi(r)$ satisfies the Poisson equation $\nabla^{2} \phi(r)-\kappa^{2} \phi(r)=-4 \pi \rho_{Q} \theta(R-r)$, where $\kappa=\sqrt{4 \pi e^{2}\left(\partial n_{e} / \partial \mu_{e}\right)_{\text {init }}}$ is the inverse of 
the TF screening length. Its solution reads as

$$
\phi(r)= \begin{cases}\left(4 \pi \rho_{Q} / \kappa^{2}\right)[1-\exp (-\kappa R)(1+\kappa R) \sinh (\kappa r) / \kappa r], & r \leq R \\ \left(4 \pi \rho_{Q} / \kappa^{2}\right)[\kappa R \cosh (\kappa R)-\sinh (\kappa R)] \exp (-\kappa r) / \kappa r, & r>R .\end{cases}
$$

Figure 2 exhibits a typical example of the electron screening at $R \sim 3 \mathrm{fm}$. When the initial chemical potential of electrons $\mu_{e, \text { init }} \gg e \phi$, as in Fig. 2 , the linear TF approximation can be safely used. For $R \lesssim 10 \mathrm{fm}$, it turns out that $e \phi / \mu_{e, \text { init }} \lesssim 0.1$. Within the confines of this approximation, $\Delta E_{e}(R)=Z \mu_{e, \text { init }}$.

We then calculate the electrostatic energy $E_{C}(R)$ using Eq. (6). The result is

$$
E_{C}(R)=\left(2 \pi^{2} \rho_{Q}^{2} / \kappa^{5}\right)\left\{-3+(\kappa R)^{2}+\exp (-2 \kappa R)\left[3+6 \kappa R+5(\kappa R)^{2}+2(\kappa R)^{3}\right]\right\} .
$$

Note that, in the limit of electron uniformity $(\kappa R \rightarrow 0), E_{C}(R) \rightarrow 3 Z^{2} e^{2} / 5 R$.

The surface tension $\sigma$, being poorly known, has been taken from the Fermi-gas model for a quark matter droplet in vacuum. ${ }^{8)}$ Due to the reduction in quark density of states arising from the droplet surface, the surface tension $\sigma=\left(3 / 4 \pi^{2}\right)\left(m_{u} \mu_{u}^{2}+m_{d} \mu_{d}^{2}\right)$ occurs for $\mu_{q} \gg m_{q}(q=u, d)$. Since $\mu_{u} \sim 500$ $\mathrm{MeV}, \mu_{d} \sim 600 \mathrm{MeV}$, and $m_{u} \sim m_{d} \sim 10 \mathrm{MeV}$, the value of $\sigma$ is roughly estimated as $\sigma \sim 10 \mathrm{MeV}$ $\mathrm{fm}^{-2}$. The Fermi-gas description of finite quark matter as adopted here, however, is problematic for a small baryon number inside the bag $\left.(\lesssim 5) .{ }^{9}\right)$ Nevertheless, we expect such a description to be valid, since the baryon number of a virtual droplet moving under the potential barrier is $\gtrsim 10$ (typically $\sim 100)$.

Finally, we determine the number densities $n_{u}$ and $n_{d}$. As a result of deconfinement, these are re-

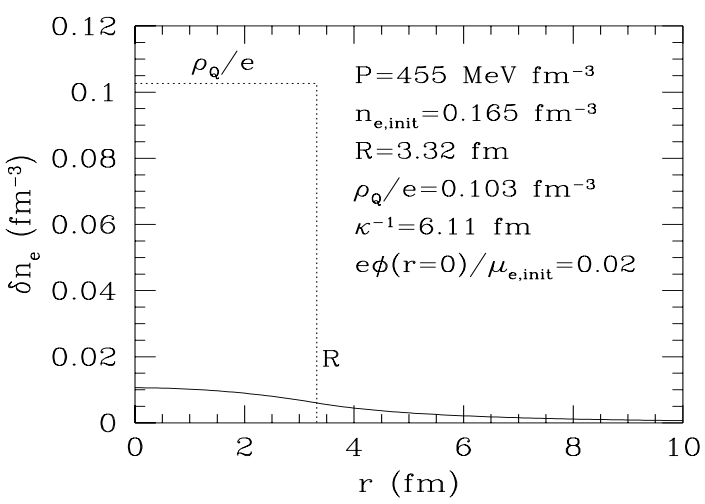

Fig. 2. Deviation $\delta n_{e}$ of the electron number density from the uniform one $n_{e, \text { init }}$ due to the TF screening on the excess droplet charge of density $\rho_{Q}$ and of radius $R$ at $P=455$ $\mathrm{MeV} \mathrm{fm}{ }^{-3}$. See the text for the definition of $r, \kappa, \mu_{e \text {,init }}$, and $\phi$. lated via $n_{u} / n_{d}=\left(1+x_{\text {init }}\right) /\left(2-x_{\text {init }}\right)$. Another relation comes from the pressure equilibrium between quark and nuclear matter,

$$
P_{Q}=P_{N}-\frac{\Delta E_{e}(R)}{4 \pi R^{3}},
$$

where $P_{Q}=\left[\epsilon_{Q}\left(n_{u}, n_{d}\right)-4 B\right] / 3$ is the quark pressure, and $P_{N}=P-\epsilon_{e}\left(n_{e, \text { init }}\right) / 3$ is the nucleon pressure. As long as $\sigma \propto n_{q}^{2 / 3}$, the surface tension contributes nothing to the 
pressure equilibrium (8). In Eq. (8) the Coulomb pressure arising from $E_{C}(R)$ has been ignored since it is negligibly small. $n_{u}$ and $n_{d}$ are thus independent of $R$.

We next estimate the effective mass $M(R)$ from the kinetic energies of nucleons and of electrons. We obtain the nucleon kinetic energy by solving the nucleon continuity equation with the boundary condition at the droplet surface ${ }^{5)}$ as $2 \pi \epsilon_{N}\left(n_{\text {init }}, x_{\text {init }}\right)\left(1-n_{b, Q} / n_{\text {init }}\right)^{2} R^{3} \dot{R}^{2}$. The electron kinetic energy, which is calculated from the electron continuity equation as $<2 \pi \epsilon_{e}\left(n_{e, \text { init }}\right)\left(\rho_{Q} / n_{e, \text { init }} e\right)^{2} R^{3} \dot{R}^{2}$, proves negligible since $\epsilon_{N} \sim 1000 \mathrm{MeV} \mathrm{fm}^{-3} \gg \epsilon_{e} \sim 40$ $\mathrm{MeV} \mathrm{fm}{ }^{-3}$. We thus obtain

$$
M(R)=4 \pi \epsilon_{N}\left(n_{\text {init }}, x_{\text {init }}\right)\left(1-\frac{n_{b, Q}}{n_{\text {init }}}\right)^{2} R^{3} .
$$

We turn to the evaluations of the time $\tau$ required to form a single droplet. By calculating not only the energy $E_{0}$ for the zeroth bound state around $R=0$ but also the corresponding oscillation frequency $\nu_{0}$ and probability of barrier penetration $p_{0}$ from the Lagrangian (3) in the WKB approximation, ${ }^{10)}$ the values of $\tau$ have been obtained as $\tau=\left(\nu_{0} p_{0}\right)^{-1}$. With increasing pressure from the transition point $P_{0} \cong 445 \mathrm{MeV} \mathrm{fm}^{-3}$, the potential barrier is lowered for fixed $\sigma$ as the solid lines in Fig. 3 exhibit. Since this barrier controls the underbarrier action $S_{0}$ between the classical turning points and hence the probability $p_{0}=$ $\exp \left(-2 S_{0} / \hbar\right)$, the nucleation time $\tau$ calculated for $\sigma=5,10,15$, and $20 \mathrm{MeV} \mathrm{fm}^{-2}$ shows an exponential $P$ dependence in the overpressure regime, as illustrated in Fig. 4. The oscillation

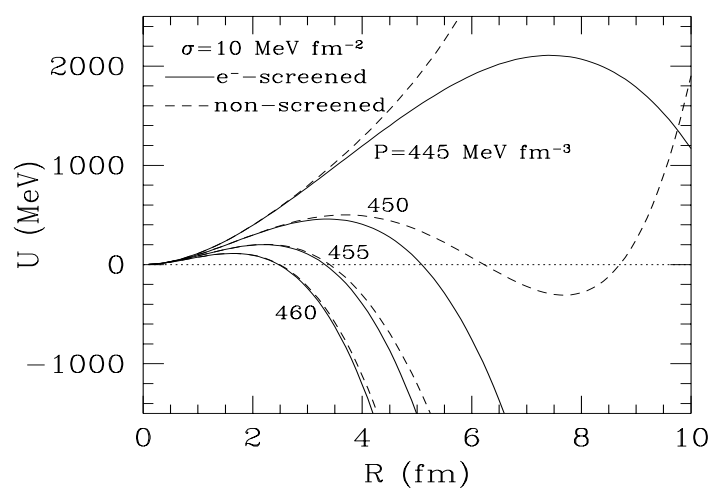

Fig. 3. Potentials for the formation of a droplet with the surface tension $\sigma=10$ $\mathrm{MeV} \mathrm{fm}^{-2}$ at $P=445,450,455$, and 460 $\mathrm{MeV} \mathrm{fm}{ }^{-3}$. The solid lines are the results for the electron-screened droplet and the dashed lines are those for the non-screened droplet.

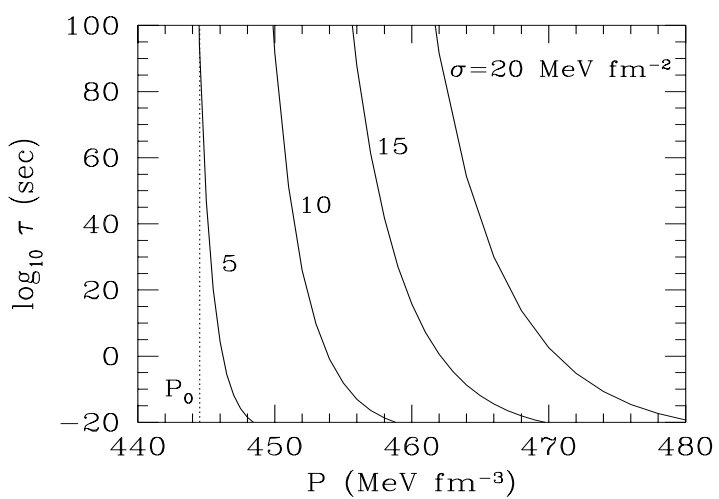

Fig. 4. Time for the formation of a single droplet with the surface tension $\sigma=$ $5,10,15$, and $20 \mathrm{MeV} \mathrm{fm}^{-2}$ as a function of pressure. $P_{0}$ denotes the transition point. 
time scale is naturally $\nu_{0}^{-1} \sim 10^{-23}$ sec. The energy $E_{0}$ is mostly comparable to the barrier height $\sim 100 \mathrm{MeV}$. Nevertheless, the WKB approximation is expected to be useful because the WKB analysis of tunneling for the Lagrangian having no Coulomb energy but otherwise the same $R$ dependence as Eq. (3) agrees well with the fully quantum analysis. ${ }^{11)}$ It is important to note that the formation time of the first droplet anywhere in a neutron star is $\tau / N$, where $N$ is the number of virtual centers of droplet formation in the star. $N$ is uncertain, but is at most the total baryon number in the star (typically $\sim 10^{57}$ ).

Finally, we give some astrophysical implications. The nuclear equation of state of intermediate stiffness used here allows a neutron star to have the central density higher than the transition point $\simeq 5 n_{0} \cdot{ }^{12}$ ) Once the central pressure reaches the pressure giving a realistic value of $\tau / N$ during the spin-down or accretion, a droplet of $u$ and $d$ quarks may appear in the nuclear matter. We can see from Fig. 3 that the electron screening, without which $E_{C}$ $\left(\propto R^{5}\right)$ dominates $U(R)$ for large $R$, prevents the droplet from remaining finite for $R \lesssim 10$ fm. For $R \gg \kappa^{-1}, E_{C}$ behaves as $E_{C} \propto R^{2}$ and $E_{C} \lesssim 4 \pi \sigma R^{2}$, hence $U(R)<0$. The droplet may thus expand into bulk matter with the speed of sound, induced by the density jump due to deconfinement. The resultant bulk quark matter occupying the region $P \gtrsim P_{0}$ may change into three-flavor quark matter via weak interactions in a time scale of $10^{-9}$ sec. $^{13)}$ As long as three-flavor quark matter is more favorable than nuclear matter, the region of $u, d$, and $s$ quarks may spread ${ }^{13)}$ with a release of chemical energy which amounts to $\sim 100 \mathrm{MeV}$ per baryon as estimated from Fig. 1(a) and turns immediately into thermal energy. This implies that the mixed phase consisting of quark and nuclear matter ${ }^{1) 2}$ is unlikely to occur. The resulting star could be stable, ${ }^{14}$ ) so the thermal energy deposited in the star $\sim 10^{52}$ ergs might be a possible origin of a $\gamma$-ray burst. ${ }^{15)}$

In summary, we have found that a droplet of $u$ and $d$ quarks, if it appeared in a neutron star, would develop into bulk matter due to the electron screening effects. The effect of muons, which was neglected above, makes nuclear matter more stable $\left(P_{0} \approx 465 \mathrm{MeV}\right.$ $\mathrm{fm}^{-3}$ ) and shortens the TF screening length by $\simeq 1 \mathrm{fm}$. We have confirmed that such a change makes no important difference. In order to make better estimates, we should examine dissipation effects on the nucleation, which may depend on the superfluidity of nuclear matter (e.g., Ref. 16)) and control the crossover temperature from classical ${ }^{17)}$ to quantum nucleation, the roles of hyperons in the composition of matter before and after deconfinement, and the poorly-known interfacial properties, the effects of curvature from the quark side being shown to significantly destabilize the droplet. ${ }^{18)}$ In addition to recent low-temperature experiments, ${ }^{19)}$ astrophysical dense matter such as neutron star matter of temperature $\lesssim 0.1 \mathrm{MeV}$ may give us examples of quantum nucleation at first-order phase transitions. 
This work was supported in part by Grants-in-Aid for Scientific Research provided by the Ministry of Education, Science, and Culture of Japan through Research Grants Nos. 05243103, 07CE2002, and 4396.

\section{References}

1) N.K. Glendenning, Phys. Rev. D46 (1992), 1274.

2) H. Heiselberg, C.J. Pethick, and E.F. Staubo, Phys. Rev. Lett. 70 (1993), 1355.

3) G. Baym, Nucl. Phys. A590 (1995), 233c.

4) E. Witten, Phys. Rev. D30 (1984), 272. C. Alcock, E. Farhi, and A. Olinto, Astrophys. J. 310 (1986), 261.

5) I.M. Lifshitz and Yu. Kagan, Zh. Eksp. Teor. Fiz. 62 (1972), 385 [Sov. Phys. JETP 35 (1972), 206].

6) H. Heiselberg, Phys. Rev. D48 (1993), 1418.

7) F.J. Dyson, Ann. Phys. (N.Y.) 63 (1971), 1.

8) M.S. Berger and R.L. Jaffe, Phys. Rev. C35 (1987), 213; Phys. Rev. C44 (1991), R566.

9) E. Farhi and R.L. Jaffe, Phys. Rev. D30 (1984) 2379.

10) T. Nakamura and S. Takagi, Mod. Phys. Lett. B9 (1995) 591.

11) T. Nakamura, Y. Kanno, and S. Takagi, Phys. Rev. B51 (1995), 8446.

12) M. Prakash, T.L. Ainsworth, and J.M. Lattimer, Phys. Rev. Lett. 61 (1988), 2518.

13) A.V. Olinto, Phys. Lett. B192 (1987), 71.

H. Heiselberg, G. Baym, and C.J. Pethick, Nucl. Phys. B (Proc. Suppl.) 24B (1991), 144.

14) A. Rosenhauer et al., Nucl. Phys. A540 (1992), 630.

15) K.S. Cheng and Z.G. Dai, Phys. Rev. Lett. 77 (1996), 1210.

16) Ø. Elgarøy, L. Engvik, M. Hjorth-Jensen, and E. Osnes, Phys. Rev. Lett. 77 (1996), 1428.

17) J.E. Horvath, O.G. Benvenuto, and H. Vucetich, Phys. Rev. D45 (1992), 3865.

M.L. Olesen and J. Madsen, Phys. Rev. D49 (1994), 2698.

18) J. Madsen, Phys. Rev. Lett. 70 (1993), 391.

19) T. Satoh et al., Phys. Rev. Lett. 69 (1992), 335.

J.P. Ruutu et al., Phys. Rev. Lett. 77 (1996), 2514. 